\section{Die Wirkung der Abführmittel und ihre Verwendung in der ärztlichen Praxis.}

Von Prof. Franz Mäller in Berlin.

Das experimentelle Studium der Wirkung von Abführmitteln, d. h. von Arzneistoffen, die die Beförderung des Darminhalts beschleunigen oder herbeiführen, ist durch Verwendung der Röntgentechnik in ein neues, ich möchte sagen der praktischen Verwendungsairt mehr entsprechendes Stadium getreten. Anschließend an $\mathrm{C}$ an n on, hat R. Mag nus mittels Röntgenstrahlen an mit Wismutbrei gefütterten Katzen die Fortbewegung des Darminhalts sehr eingehend untersucht. Es gelingt so, die Steigerung der Magenperistaltik, die Zahl der Oeffnungell des Pylorus, die Fortbewegung des Speisebreies im Dünndarm, das erste Auftreten des Wismutschattens im Dickdarm und den weiteren Weg des Darminhalts in dem Dickdarm zugleich mit dem Auftreten von peristaltischen Wellen bis zum Uebertritt in den Mastdarm und bis zur Entleerung zu verfolgen.

Der normale Darm zeigt fortschreitende rhythmische Kontraktionen der Ring- und Längsmuskulatur, durch die der Darminhalt durcheinandergemischt und fortbewegt wird, die Pendelbewegungen, ferner reflektorische, durch Reize hervorgerufene Einschnürungen und tomische Kontraktionen, die den Darminhalt weiterschieben und schlieBlich entleeren, die Peristaltik, und endlich plötzlich einsetzende, lange Kontraktionswellen, die Rollbewegungen. Im proximalen Teil des Dickdarms sieht man auch ohne therapeutische Beeinflussung oder ohne krankhafte Veränderungen antiperistaltische Bewegungen zur Bauhinschen Klappe und sogar darüber hinaus bis ins lleum hinein. Der zwischell Ring- und Längsmuskulatur liegende Auerbachsche Plexus beherrscht die Darmbewegungen zusammen mit dem parasympathischell Vagus und Pelvikıs, deren Reizung die Darmbewegungen anregt, und dem sympathischen Splanchnikus, dessen Reizung die Darmbewegungen hemmt.

Gemeinhin faßt man unter "Abführmitteln" nach der von Schmiedeberg stammenden Trennung die im ganzen Darm die Resorption erschwerenden und damit den Kot flüssig erhaltenden Stoffe, die abführenden Salze oder Kolloide, und die bestimmte Darmabschnitte erregenden pflanzlichen Abführmittel zusammen. Wir wissen aber durch die auch aus Magnus' Institut stammenden Untersuchungen von Le Heux u. A., daB der Darm selbst einen seine Tätigkeit erregenden Stoff produziert, das $\mathrm{Cholin}$. Es findet sich in der AuBenflüssigkeit eines überlebend in Tyrode-Lösung (einer modifizierten Ringerlösung) arbeitenden Darmstückes und gehört zur Gruppe der parasympathischen Gifte, die die Vagusendigungen erregen. Je nachdem der Darm Cholin enthält oder durch Auswaschen von ihm befreit ist, wirkt das die parasympathischen Nervenendigungen inı allgemeinen lähmende, also gegenüber Cholin antagonistische A t ro pi il beruhigend oder - da es auch den Auerbachschen Plexus erreg - anregend. Ein Teil der „Darmwirkung“ des Atropins ist demnach eine indirekte, indem nur Erregungsgifte beseitigt werden.

Man verwendet bekanntlich Atropin in Form der Belladonnapräparate bei Darmspasmen und spastischer Obstipation, entweder in Form der Suppositorien (0,02-0,05 Extract. Belladonnae auf 2,0 von Ol. Cacao) oder per os, etwa als die Trousseauschen Pillen (Fol Bellad. et Extr. Bellad. àa 0,3, Succ. et pulv. Liquirit. q. s. ut $f$ pil. 30) oder in Form der Gelonida stomachica mit je 0,005 oder der Gelonida stomachica fortiora mit je 0,01 Extr. Belladonnae oder kombiniert mit pflanzlichen Abführmitteln, wie Extractum Rhei compositum. Auch die Kombination mit $\mathrm{Pa}$ a verin in Pillenform hat sich nach folgendem Rezept vielfach bei Mischformen von atonischer und spastischer Obstipation bewährt.

$$
\begin{aligned}
& \text { Rp. Extract. Belladonnae } \quad 0,3 \text { oder } 0,6 \\
& \text { Papaverin. hydrochlor. } 1,2 \\
& \text { Radix et Succus Liquir. aa } 0,6 \\
& \text { M. f. Pil. } 30 \text {. } \\
& \text { S. 3mal taglich I Pille zu nehmen. }
\end{aligned}
$$

Da die normale Automatie des Darmes durch Cholin und wahrscheinlich andere, noch nicht im einzelnen bekannte' Hormone reguliert wird, müssen auf die parasympathischen Nervengebiete des Darmes im Sinne der Beseitigung einer Muskelerschlaffung, d. h. tonussteigernd wirkende Stoffe gelegentlich auch abführend wirken. Sie beseitigen eben die "Atonie". Das ist neuerdings im Utrechter Institut bei verschiedenen Arten von experimentell hervorgerufenen Darmlähmungen für das Cholin im Röntgenogramm und am überlebenden Darmstück nachgewiesen worden. Der Praktiker, besonders der Chirurg, sucht seit langer Zeit nach einem Mittel, das die bei Bauchoperationen und schon nach der Chloroformnarkose auftretende Darmlähmung sicher zu beseitigen gestattet. Man hat das von dem "Hormonal" und dem angeblich albumosefreien "Neohormonal" behauptet, ohne $\mathrm{da} B$ darüber bisher Einstimmigkeit herrscht. Störend ist, da derartige Stoffe, die, um sicher zu wirken, intravenös in jiziert werden, als allgemeine parasympathische Gifte durch Vagusrei zung die Schlagfolge des Herzens meist verlangsamen und den Blutdruck oft vorübergehend bis zu gefährlicher Tiefe senken. Die neueren Untersuchungen über die Wirkung des Cholins bei Darmatonie versprechen in dieser Hinsicht einen Fortschritt. (Bei der Katze wirkt intravenöse Injektion von $35 \mathrm{mg}$ Cholinchlorid pro Kilogramm, wenn sie nicht allzu schnell gemacht wird, nicht allgemein giftig und vermag doch schon die postoperative Magendarmlähmung zu beseitigen.)

Wenden wir uns jetzt zu den eigentlichen "Abführmitteln", so wirken die die Peristaltik bestimmter Darmabschnitte anregenden pflanzlichen Abführmittel meist auf den Dickdarm. Dagegen erregt die nach Eingabe von Ri zi nus öl im Darm entstehende Rizinolsäure den Dünndarm und gelangt kaum noch in den Dickdarm. Dem entspricht die Erfahrung der Praxis, da $B$ man bei Verdacht einer Appendizitis sich hüten muB, Rizinusöl zu verordnen, da die Wirkung eben hauptsächlich im Dünndarm und erst nach Verseifung zu Rizinolseife eintritt, und anderseits, daB man Rizinusöl bei Schwangeren verwenden darf, ohne Gefahr zu laufen, daß durch eine Dickdarmreizung mit gleichzeitig eintretender Hyperämie in den Gebieten der Vena mesenterica inferior auch der Uterus zu Kontraktionen gereizt und Abort herbeigeführt wird - eine Gefahr, die bei allen auf den Dickdarm wirkenden Abführmitteln sehr wohl besteht. Sind doch derartige, drastisch wirkende Stoffe Abortiva. Das Rizinusöl hat gegenüber den ähnlich, aber stärker wirkenden, den Dünndarm erregenden Mitteln, wie Krotonöl, Jalappenharz, Koloquinten und Podophyllin, auBerdem den Vorzug, weder Gastroenteritis noch auch Nierenreizung zu verursachen. Rizinusöl ist also für einmalige gründliche Entleerung sehr zu empfehlen.

Die den Dickdarm erregenden Drogen enthalten sämtlich Anthrazenderivate, sogenannte Emodine, die aus in den Pflanzen enthaltenen Glukosiden durch hydrolytische Spaltung und Oxydation im Darm ent stehen. Da die Mittel erst nach Eintritt in den Dickdarm wirksam werden, dauert es immer mehrere Stunden, bis die Peristaltik, oft unter recht störenden Kolikschmerzen und Tenesmen, erregt wird und flüssige Entleerungen erfolgen.

Das zur Zeit fast billigste derartige Mittel sind die Sennes. blätter, von denen ein Teelöffel auf eine Tasse kalten Wassers, 12 Stunden stehen gelassen, einen stark wirkenden Tee abgibt. Sie sind auch in den heute als veraltet zu bezeichnenden Species laxantes und in dem Kurellaschen Brustpulver (Pulvis liquiritiae compositus) enthalten. Der früher viel gebrauchte Selınainfus und das Electuarium e Senna sind wegen der teueren Herstellungsart nicht mehr zu empfehlen. Sennesblätter verursachen außerdem oft recht starke kolik. artige Schmerzen.

Faulba um rinde ist das einzige aus in Deutschland heimischen Pflanzen stammende pflanzliche Abführmittel. Man ist dazu übergegangen, die Rinde im großen zu kultivieren. Alle anderen, Oxyanthrachinone im Darm liefernden Drogen kommen aus dem Ausland und sind daher entsprechend teuer geworden. Man wird daher Faulbaumrinde vor Senna, Rhabarber, Aloe und Cascara sagrada möglichst bevorzugen.

Viel gebraucht werden, wenn man nicht einfach Rhabarberpulver geben kann, die Pilula e Rhe i nach der Berliner Magistralformel (Rhizoma Rhei pulv. 6,0, Glycerini 2,2 auf 30 Pillen). Nicht viel teurer sind die Pilulae aloëticae FMB. (Aloës pulv. 3,0, Saponis Jalap. 1,8, Spiritus 0,4 auf 30 Pillen), erheblich teurer dagegen die Pilulae laxantes fortes FMB.' (Extract. Colocynthidis 0,24 Extract. Aloës 2,4, Resina Jalap. et Sapo medic. $\bar{a} \bar{a}$ 1,2, Spiritus 0,2 auf 30 Pillen).

Wie jetzt so häufig, ist ein künstlich, synthetisch hergestellter Stoff billiger als das Naturprodukt. Das I s tizin (B a y e r) ist ein derartiges Dioxyanthrachinon, das in Tablettenform zu 0,15 die gleiche Wirkung entfaltet wie die genannten pflanzlichen Stoffe

In zahlreichen abführenden Spezialitāten findet sich als wirksamer Stoff Phenolphthalei in, das zuerst als "Purgen" in den Handel kam. Es ist ein mildes und sicheres Abführmittel, hat aber den nicht zu unterschätzenden Nachteil, daß es häufig Nierenreizung hervorruft. Die zahlreichen Laxinpillen und Laxinkonfekte sind daher zumal bei dauerndem Gebrauch, durchaus nicht immer ungefährlich

Auch Eingabe von Sch wefel erregt infolge Reduktion durch die Darmbakterien und die EiweiBstoffe der Dünndarm- und Dickdarmschleimhaut die Darmperistaltik. Die Magenschleimhaut enthält keine den Schwefel reduzierenden Stoffe, die Wirkung beginnt daher bei fortdauernder Bildung kleiner Mengen von Schwefelwasserstoff erst im Dünndarm. Da die hohe Kohlensäurespannung des Darminhalts die Bildung von Schwefelalkalien, die die Darmschleimhaut anätzen und entzündlich reizen könnten, verhindert, kommt es nach Eingabe von Schwefel niemals zu gefährlicher Reizung, nicht einmal zu Diarrhoe. Die Darmentleerungen werden vielmehr nur breiig, ohne daß der Patient durch Kolikschmerz belästigt wird.

Das nicht bloB in der Kinderpraxis mit Erfolg bei akuten Darmkatarrhen verwendete $\mathrm{Kalomel}$ erzeugt in Mengen von 0,3-0,4 beim Erwachsenen, von 0,01-0,03 dreimal täglich bei Kindern starke Beschleunigung der Dünndarmentleerung und Dickdarmpassage, wie kürzlich inı Magnusschen Institut gefunden wurde. Ob außerdem, wie man bis vor kurzem annahm, eine Erregung der Drüsensekretion und .Hemmung der Wasserresorption aus dem Dünndarm mitspielt, ist neuerdings fraglich geworden. Bekanntlich muB die Wirkung des Kalomels prompt erfolgen, das Mittel darf nicht im Darm zurückbleiben, weil sonst die Gefahr der Quecksilbervergiftung besteht. Erwünscht ist die hohe desinfizierende Kraft gerade bei infektiösen Darmerkrankungen. Man wird Kalomel aber, bei gleichzeitig bestehender Nierenerkrankung oder bei Gefahr der Darmlähmung und jeder Störung der Darmpassage zu vermeiden haben. 
Die mineralischen Abführmittel, die abführenden Salze, verdanken ihre Wirkung rein physikalisch-chemischen Erscheinungen. Die durch tote Membranen (Schweinsblase, Gelatineplatten usw.) schwer diffundierenden Salze werden auch vom Darm aus schwer resorbiert. Sie haben ein hohes Wasseranziehungsvermögen und halten das Lösungswasser im Darm zurück. Zu dieser Gruppe gehören von den Kationen Magnesium, von den Anionen vor allem Phosphorsäure und Schwefelsäure. Trocken eingenommenes Glaubersalz (Natriumsulfat) und Bittersalz (Magnesiumsulfat) erzeugen Flüssigkeitsansammlung im Darm, Abwanderung von Wasser aus den Geweben und dem Blut in den Darm. Die Entleerung erfolgt nach 8-10 Stunden. Die konzentrierte Salzlösung reizt gleichzeitig die Darmdrüsen zu vermelirter Sekretion und bewirkt so auch Zunahme der Gallenabsonderung. Die abführende Wirkung wird nach Einnahme der festen Salze oder von konzentrierten Lösungen um so stärker sein, je wasserreicher das Blut und die Gewebe sind. So erklärt sich die günstige Wirkung beim Oedematösen.

Werden aber die Sulfate oder Phosphate in verdünnterer Lösung getrunken (von $5 \%$ an abwärts), so wird ein Teil des Lösungswassers im Dünndarm resorbiert, erzeugt Diurese, und nur der nicht resorbierte Rest gelangt.schnell in den Dickdarm, wo er kraft seines Wasserverbindungsvermögens die Eindickung des Darminhalts verhindert. Die Wirkung erfolgt in diesem Falle innerhalb der ersten zwei Stunden und ist unabhängig vom Wassergehalt des Blutes und der Gewebe. So haben wir uns einen Teil der Wirkung der abführenden Quellen zu erklären, bei der aber die Zunahme der Blutfülle im Darm- und Pfortadergebiet und der Gallensekretion nicht zu vernachlässigen ist.

Die Untersuchungen von Wiech ow ski und seinen Mitarbeitern Starkenstein und Stransky über die Wirkung des Karlsbader Wassers auf den Mineralstoffhaushalt haben ferner gezeigt, daß wesentliche Aenderungen im Mineralbestand des Organismus beim Kaninchen durch Ersatz des gewöhnlichen Trinkwassers durch Mineralwasser herbeigeführt werden können. Nicht nur der absolute Gehalt an Kationen, sondern auch das Verhältnis von Kalium und Natrium zum Magnesium und Kalzium, sowie anderseits von Chlor zu Phosphat wird hierdurch beeinfluBt. Unter dem Einfluß des sulfatreichen Karlsbader Wassers werden die Phosphate in höherem MaBe im Organismus zurückbehalten. Da wir wissen, eine wie große Bedeutung die Phosphorsäure für zahlreiche Fermentreaktionen des Organismus hat, wird es verständlich, daB die mit Karlsbader Wasser oder künstlichen Sulfatgemischen getränkten Tiere sich gegenüber Entzündungen, Temperaturänderungen („Fieberbereitschaft") und anderem anders verhalten als zuvor. Es sei ferner andeutungsweise - da nicht direkt hierher gehörig - darauf hingewiesen, daß Aenderungen des Salzmilieus im Phosphatgehalt erhebliche Aenderungen im Verlauf der Zuckergärung hervorbringen, wie $\mathrm{Paul} M$ a y e r ganz kürzlich gezeigt hat - ein Hinweis darauf, wie man vielleicht die Wirkung der Karlsbader Kur bei bestimmten Diabetesformen zu deuten hat. Wir befinden uns hier im Anfang von für die Erkenntnis der Mineralwasserkuren bedeutungsvollen Forschungen, die zum ersten Male einen exakten und der experimentellen Prüfung zugänglichen Beweis dafür geliefert haben, daB durch eine längere Trinkktur mit sulfatreichen Quellen eine erhebliche Aenderung im Mineralstoffwechsel und -gehalt des Organismus hervorgebracht wird. Leider ist durch die Verteuerung des Transportes und der Flaschenpreise die Beschaffung, von Karlsbader Wasser nur noch wenigen Begäterten möglich. Man wird sich mit dem Gebrauch des künstlichen Karlsbader Salzes, einem Gemisch aus 44 Teilen Natriumsulfat, 2 Teilen Kaliumsulfat, 36 Teilen Natriumbikarbonat und 18 Teilen Kochsalz, begnügen und durch Lösen etwa eines EBlöffels in warmem Wasser einen gewissen Ersatz für Mühlbrunnen oder Sprudel schaffen müssen. Wie allgemein bekannt, trinkt man das Wasser früh morgens nüchtern und nimmt nach Bewegung das Frühstück erst eine Stunde. später ein. Uebrigens wirkt oft schon das Trinken eines Glases reinen Wassers auf nüchternen Magen abführend!

Einen Ersatz für die gleichfalls sehr teuer gewordenen „Bitterwässer" schafft man sich durch Auflösen von 1 bis 2 EBlöffeln Bittersalz in warmem Wasser. Auch Magnesia us ta und Magnesium! perhydrol (Tabletten Merck zu 0,5) wirken abführend. Das früher viel gebräuchliche Seignettesalz, das in dem abführenden Brausepulver und im Wiener Trank enthalten ist, ist gleichfalls zil teuer, geworden, als daß man es noch empfehlen sollte.

Neben den schwer resorbierbaren Salzen tritt die Verwendung von durch Quellung Wasser anziehenden Stoffen, wie AgarAgar, dem Hauptbestandteil der Regulinpillen, an Bedeutung stark zurück.

Die Verordnung von Abführmitteln bei atonischer, chronischer Obstipation ist gegenüber der Regelung der Verdauung durch zweckentsprechende Diät sehr mit Recht in den Hintergrund getreten. Oft genügt die Umschaltung von $\mathrm{zu}$ konzentrierter Fleischnahrung, zumal beI WeißbrotgenuB, auf ein vegetarisches Regime mit reichlich zellulosehaltigen Speisen, wie Gemüsen, Salat, Obst und Schwarzbrot oder Schrotbrot, um die Darmträgheit zu beheben. Oft ist auch die Verordnung regelmäBiger Körperbewegung, Gymnastik, Sport, Massage und Hydrotherapie empiehlenswerter als dauernder Gebrauch von abführenden Medikamenten. Gerade bei der chronischen Obstipation muB der Arzt weitgehend individualisieren und den ganzen Organismus, nicht bloB den Darmkanal behandeln.

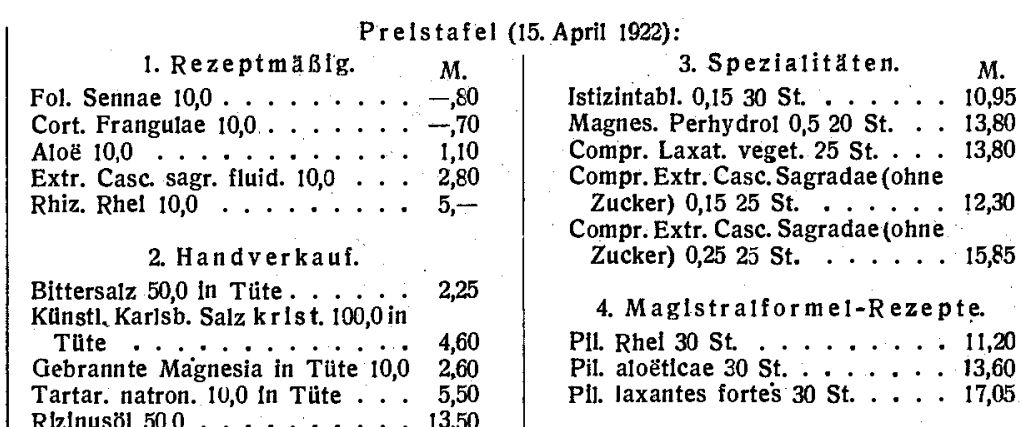

